\title{
Tunneling Images of Atomic Steps on the Sil111)7×7 Surface
}

\section{Citation}

Becker, R. S., J. A. Golovchenko, E. G. McRae, and B. S. Swartzentruber. 1985. Tunneling Images of Atomic Steps on the Si(111)7×7 Surface. Physical Review Letters 55, no. 19: 2028-2035. doi:10.1103/physrevlett.55.2028.

\section{Published Version}

doi:10.1103/PhysRevLett.55.2028

\section{Permanent link}

http://nrs.harvard.edu/urn-3:HUL.InstRepos:29407044

\section{Terms of Use}

This article was downloaded from Harvard University's DASH repository, and is made available under the terms and conditions applicable to Other Posted Material, as set forth at http:// nrs.harvard.edu/urn-3:HUL.InstRepos:dash.current.terms-of-use\#LAA

\section{Share Your Story}

The Harvard community has made this article openly available.

Please share how this access benefits you. Submit a story.

\section{Accessibility}




\title{
Tunneling Images of Atomic Steps on the Si(111) $7 \times 7$ Surface
}

\author{
R. S. Becker, J. A. Golovchenko, E. G. McRae, and B. S. Swartzentruber \\ AT\&T Bell Laboratories, Murray Hill, New Jersey 07974 \\ (Received 11 July 1985)
}

Tunneling images of single atomic steps on $\mathrm{Si}(111)$ have been obtained with a tunneling microscope. The $7 \times 7$ reconstruction is observed to persist essentially undistorted right up to the steps. The position of each step coincides with a $7 \times 7$ unit-mesh edge in both the upper and lower terraces adjacent to it. Asymmetry within the $7 \times 7$ unit mesh is observed and correlated with faulting of terraces adjacent to the step. The results are described by an extension of the dimer adatom stacking-fault model of Takayanagi et al.

PACS number: $68.20 .+\mathrm{t}$

Atomic steps at crystal surfaces have an important role in chemical reactions and in the growth of crystals by molecular-beam epitaxy. Yet the microscopic structure of steps, which is basic to the understanding of these processes, is not known and is not directly accessible to any widely used surface-science technique. In the case of the $\mathrm{Si}(111) 7 \times 7$ surface, for example, it is not known whether the reconstruction observed by reflection electron microscopy ${ }^{1}$ on flat terrace areas extends unmodified right up to step edges. There is indirect evidence that the step-edge structure is not well described by simple models of unreconstructed terraces; in observations of the participation of steps in molecular-beam epitaxial growth on $\mathrm{Si}(111) 7 \times 7$ surfaces, it was noted that steps on the growing surface with outward normals along $\langle\overline{11} 2\rangle$ directions were straight, which is contrary to expectations based on simple atomic models. ${ }^{2}$ In the course of our studies of $\mathrm{Si}(111)$ surfaces using a tunneling microscope of the type recently described by Binnig et al., ${ }^{3}$ we have come across regions containing several steps and have had the opportunity to obtain high-quality tunneling images there for the first time. We show that the highresolution view of these steps sheds light on the nature of steps as well as of the $7 \times 7$ reconstruction itself.

The sample was a boron-doped, $0.05-\Omega-\mathrm{cm} \mathrm{Si}(111)$ wafer that was argon-ion sputtered and then annealed at $900^{\circ} \mathrm{C}$ in an ultrahigh-vacuum chamber. Very sharp $7 \times 7$ diffraction spots were observed by LEED after the sample cooled. The sample was then placed in the tunneling microscope where images were obtained after approximately $5 \mathrm{~h}$ when thermal drifts in the instrument had decayed to acceptable levels.

Figure 1 shows a tunneling image as a profile plot from the above sample. Two atomic steps are clearly seen running parallel to one another across the figure. The observed height of the steps from our independently calibrated instrument is $3.1 \AA$, in excellent agreement with the expected step height of $3.13 \AA$ for the $\mathrm{Si}(111)$ double-layer periodicity. The lateral distance scale is also indicated in the figure. By comparing the tunneling image with a Laue $\mathrm{x}$-ray-diffraction picture, we found that for the steps in the figure the outward normal of the riser is a $\langle 11 \overline{2}\rangle$ direction. We have also observed $\langle\overline{11} 2\rangle$-type steps, on which we comment later.

In addition to the steps in Fig. 1 one can clearly see atomic structure in the adjacent terrace regions, where oscillations in the observed height are of the order of 1 $\AA$. Since the geometric symmetry of these regions is difficult to pick out in such a profile plot, we present another picture of the region in a gray-scale format in Fig. 2. On this plot, white areas are raised and dark ones are depressed. Aside from a few defects, the image of the terrace surface is in good agreement with that first observed by Binnig et al. ${ }^{3}$ for $\mathrm{Si}(111) 7 \times 7$ surfaces. There is, however, a significant difference on which we shall comment later.

There are several new features in this picture that are quite remarkable. First we see that the $7 \times 7$ reconstruction extends right up to the atomic steps. In fact,

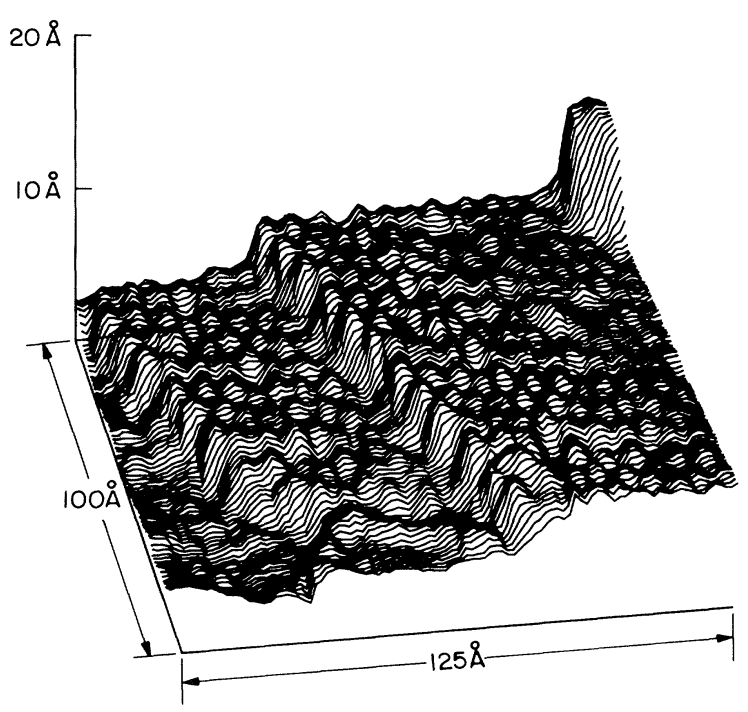

FIG. 1. A tunneling image of $\operatorname{Si}(111) 7 \times 7$ surface reconstruction on atomic steps displayed as a profile plot. The tip-to-sample bias was $-4 \mathrm{~V}$. The outward normal direction from the step riser is $\langle 11 \overline{2}\rangle$. 


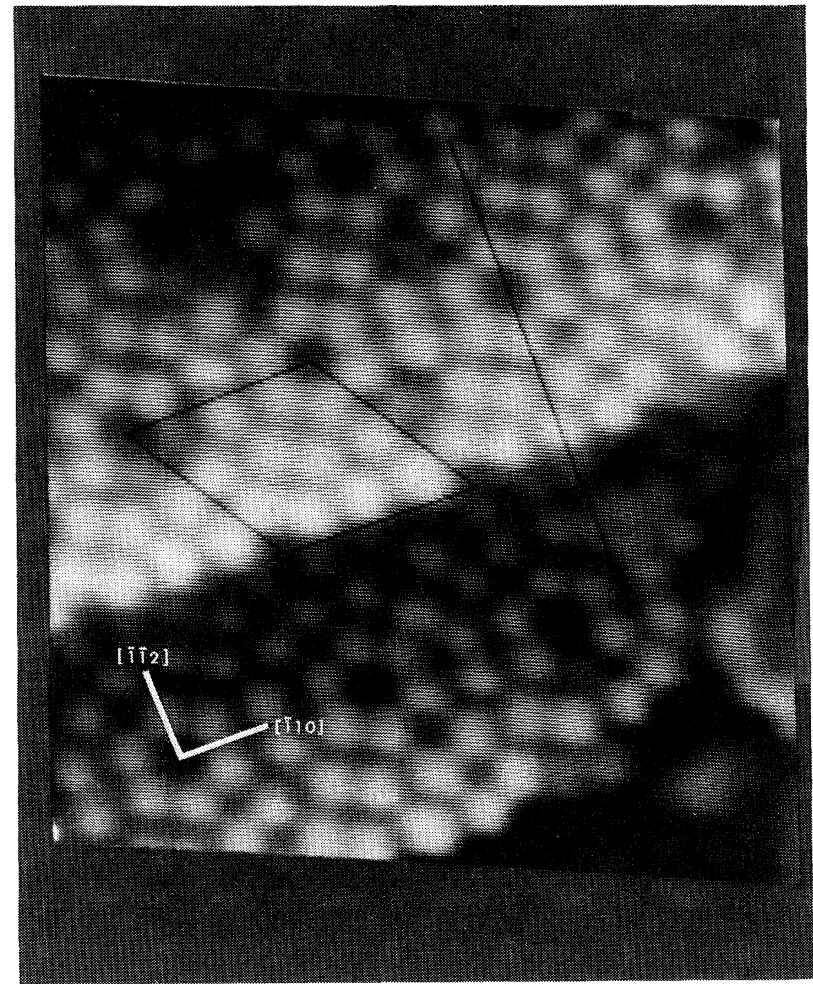

FIG. 2. The data in Fig. 1 displayed as a gray-scale plot. A unit mesh on the edge of the upper step is outlined. The length of one side of the unit mesh is $26.9 \AA$. The straight line shows the tip trajectory mentioned in the text.

the top edge of the step is observed to have a seventh-order periodicity synchronized with the reconstruction of the upper terrace. The position of each step coincides with the edge of the rhombohedral unit mesh in both the upper and lower terraces adjacent to it. The large holes associated with the corners of the unit mesh are incorporated right into the topography of the step, as can be seen in both Figs. 1 and 2 . A terrace between parallel steps of this structure must have the width of an integer number of unit meshes, i.e., two unit meshes in Figs. 1 and 2. There is a phase slip in the direction of the step edge, amounting to about $\frac{2}{7}$ of the unit-mesh side.

In addition to the properties apparent in Figs. 1 and 2 , we report a further observation related to the asymmetrical nature of the $7 \times 7$ unit mesh first noted by Binnig et al. ${ }^{3}$ We have observed this asymmetry for certain bias voltages. It is manifested as a raised base height on one of the two triangular subunits of the unit mesh [Fig. 3(a)]. In the image of the upper terrace of a $\langle 11 \overline{2}\rangle$ step, the side of the unit mesh with the lower base height is adjacent to the step. We note that this orientation is opposite to that reported by Binnig et $a l^{3}$ for an unstepped surface. Figure 3 (b) shows

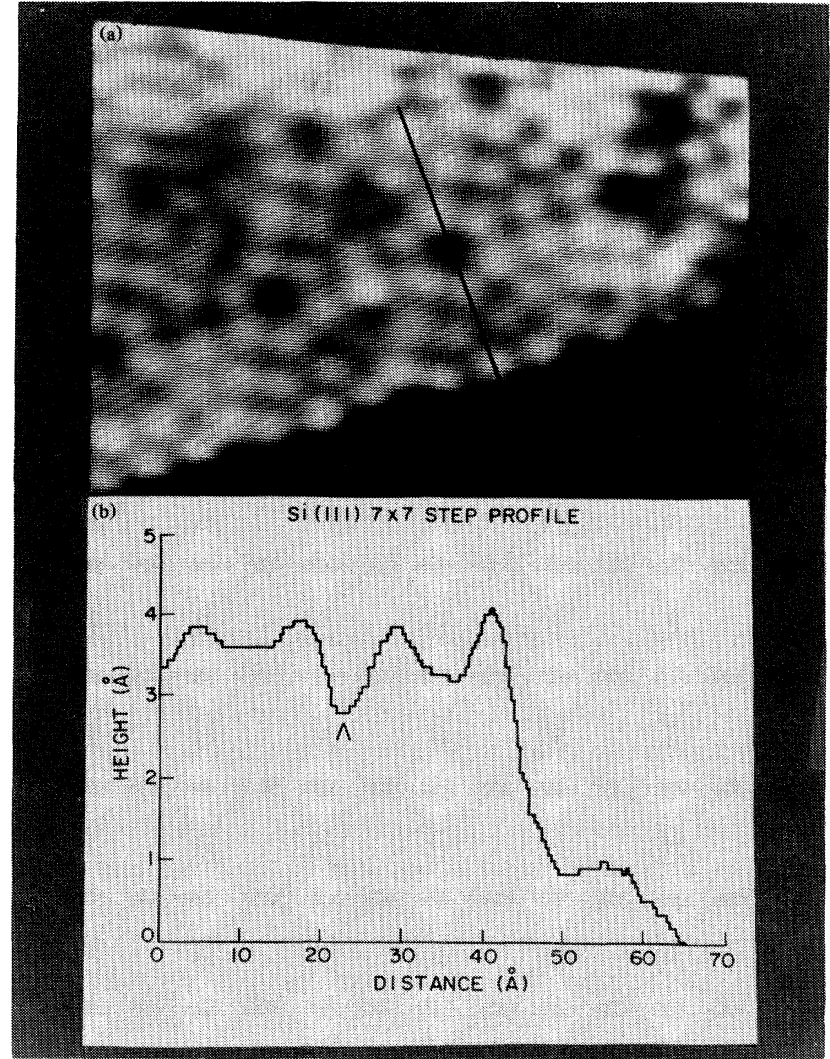

FIG. 3. (a) A tunneling image of the upper step, taken at $-3-\mathrm{V}$ bias. The asymmetry between the two halves of the unit mesh is clearly visible. (b) A plot of the data along the trajectory which bisects the different halves of a unit mesh. The arrow points to the unit-mesh corner hole.

an isolated tip-height plot across the step and along the long diagonal of a unit mesh in the upper terrace. The trajectory is shown in Figs. 2 and 3 (a). Its direction is perpendicular to the step, which is clearly seen as 3.1$\AA$ drop. There is a $0.2-\AA$ base-height asymmetry with respect to the corner hole indicated by the arrow in Fig. 3(b). A similar plot on the lower terrace of the step would show the side of the unit mesh with the high base height to be adjacent to the step.

A further observation is of steps of the $\langle\overline{11} 2\rangle$ type for which we do not display data here. The position of each step again coincides with the unit-mesh edges so that the corner holes are again incorporated in the step, but in this case the high base-height side of the unit mesh is adjacent to the upper edge of the step.

Our observations permit two general conclusions independent of any structural model. First, the persistence of the $7 \times 7$ reconstruction right up to the step implies that the reconstruction itself depends on veryshort-range, local interactions. Indeed we, as well as 
Binnig and Rohrer, ${ }^{4}$ have also observed isolated unit meshes among highly disordered neighboring regions, which also strongly supports this conclusion. The situation may be quite different from the $\mathrm{Si}(111) 2 \times 1$ surface formed by cleaving, for which it is plausible that the reconstruction does not go right up to the step. ${ }^{5,6}$ The second general conclusion is that the step is itself uniquely reconstructed. This follows from the observation that the position of the step relative to the reconstructed terraces is not arbitrary but always coincides with a unit-mesh edge in both the upper and lower terraces adjacent to it. For each type of step, the structure of the riser is related in a definite way to the $7 \times 7$ reconstruction of the terraces. In particular, the structure of the corner hole is also the structure of the step.

A more detailed discussion can be given on the basis of structural models of the $7 \times 7$ reconstruction on a flat $\mathrm{Si}(111)$ surface in which the lateral atomic arrangement (surface-projected structure) is in accord with transmission-electron-diffraction intensity data. ${ }^{7,8}$ In these models ${ }^{9-14}$ the outermost complete double layer in the unit mesh consists of two triangular subunits that are respectively faulted and unfaulted with respect to the substrate. The partial dislocations bordering the triangular subunits are reconstructed to form twelve-membered rings which are observed in tunneling microscopy as corner holes, as well as alternating dimers and eight-membered rings which are also observed as depressions. ${ }^{9}$ The origin of the tunneling-image height modulations within the triangular subunits is not completely settled, but one possibility is that they are due to adatoms., ${ }^{3,15}$ This suggestion has been incorporated in the dimer adatom stacking-fault (DAS) model due to Takayanagi, Tanishiro, Takahashi, and Takahashi. ${ }^{13}$

Figure 4 shows part of the tunneling image from Fig. 2 in comparison with a model of the same area in which the terraces have the DAS structure. The model explains the coincidence between the step edge and the unit-mesh boundaries, since any other position of the step would result in a larger number of dangling bonds. The "ping-pong balls" represent the adatoms. In the model, all but one of the adatoms are in the sites prescribed by the DAS model, namely, directly over the unique atom of a five-membered ring. Note that this is a "closed" site, i.e., there is an atom of the outermost complete double layer directly below the adatom. The remaining adatom (bottom-right ball) is on an interstitial closed site between two vacant adatom sites.

There is a good correlation between the tunneling image and the adatom positions in the model. There are slight discrepancies for the adatoms on the lower terrace adjacent to the step, but these may be an experimental artifact related to the abrupt variation of

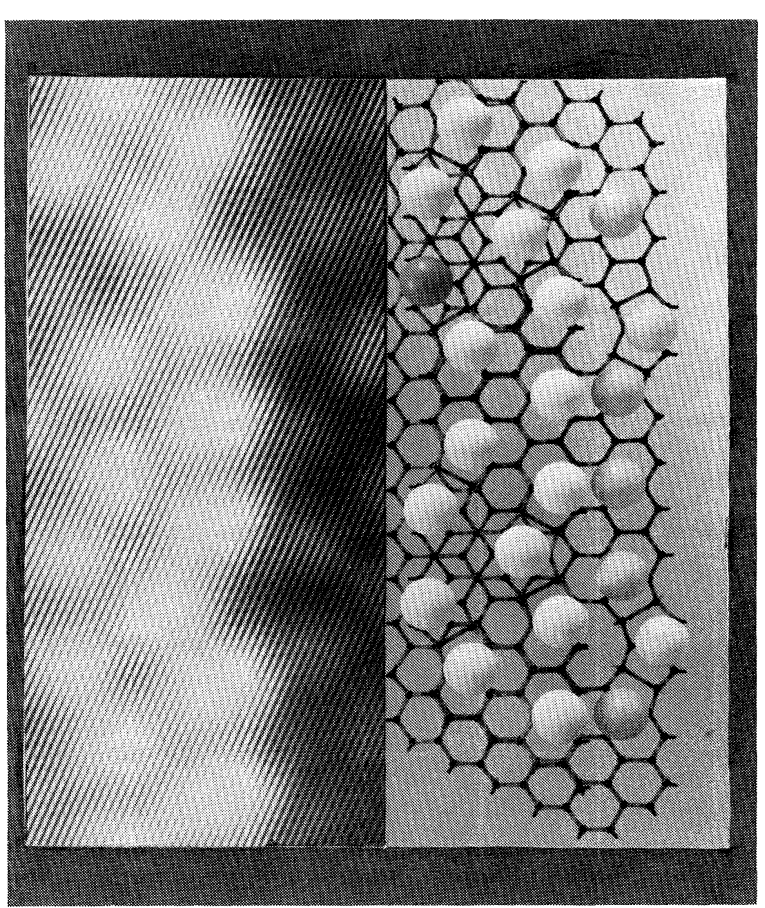

FIG. 4. A portion of data along the upper step edge (from Fig. 2) shown with a model of the same area in which the terraces have the DAS structure.

height near the step and the microscopic shape of the tip.

The defects are displayed in the tunneling image in Fig. 4: an apparent vacancy at the top right of the image (represented in the model by a dark-colored ball) and the apparent interstitial accompanied by two vacancies already referred to. The nature of these defects as well as the observed persistence of reconstruction right up to the step appear to support the adatom hypothesis.

In the model, Fig. 4, it is easy to pick out the faulted and unfaulted areas in the upper terrace near the $\langle 11 \overline{2}\rangle$ step. The faulted area is adjacent to the step, and so is identified with the triangular subunit with the lower base height in images such as that shown in Fig. 3 . In the corresponding model for the $\langle\overline{11} 2\rangle$ step, the unfaulted area of the upper terrace is adjacent to the step. Therefore, the reconstruction of the riser is the same for the two types of step.

In conclusion, tunneling images of single atomic steps on $\mathrm{Si}(111)$ have been obtained by use of a tunneling microscope. The $7 \times 7$ reconstruction is observed to persist essentially undistorted right up to the steps. The position of each step coincides with a $7 \times 7$ unit-mesh edge in both the upper and lower terraces adjacent to it. Asymmetry within the $7 \times 7$ unit mesh is observed and correlated with faulting of terraces adjacent to the step. The results are described by an ex- 
tension of the DAS model of Takayanagi et al. ${ }^{13}$

${ }^{1}$ Y. Tanishiro, K. Takayanagi, and K. Yagi, Ultramicroscopy 11, 95 (1983).

${ }^{2}$ H. C. Abbink, R. N. Broudy, and G. P. McCarthy, J. Appl. Phys. 39, 4673 (1968).

${ }^{3}$ G. Binnig, H. Rohrer, Ch. Gerber, and E. Weibel, Phys. Rev. Lett. 50, 120 (1982).

${ }^{4}$ G. Binnig and H. Rohrer, Surf. Sci. 152/153, 18 (1985).

${ }^{5}$ S. Kruger and W. Mönch, Surf. Sci. 99, 157 (1980).

${ }^{6}$ J. E. Rowe, S. B. Christman, and H. Ibach, Phys. Rev. Lett. 34, 874 (1975).

${ }^{7}$ K. Takayanagi, Y. Tanishiro, M. Takahashi, H. Motoyoshi, and K. Yagi, Electron Microsc. 2, 285 (1983).

8P. M. Petroff and R. J. Wilson, Phys. Rev. Lett. 51, 199 (1983).
${ }^{9}$ E. G. McRae, Phys. Rev. B 28, 2305 (1983), and Surf. Sci. 147, 663 (1984).

${ }^{10}$ E. G. McRae and P. M. Petroff, Surf. Sci. 147, 385 (1984).

${ }^{11}$ K. Takayanagi, J. Microsc. 136, 287 (1984).

12E. G. McRae, in The Structure of Surfaces, edited by M. A. Van Hove and S. Y. Tong (Springer, New York, 1984), Vol. 2, p. 278.

${ }^{13} \mathrm{~K}$. Takayanagi, Y. Tanishiro, M. Takahashi, and S. Takahashi, J. Vac. Sci. Technol. A 3, 1502 (1985); R. M. Tromp and E. J. Van Loenen, Surf. Sci. 155, 441 (1985).

${ }^{14}$ Tests of structural models by comparison with critical experimental evidence have been described by R. M. Tromp, Surf. Sci. 155, 432 (1985).

${ }^{15}$ Electron-diffraction evidence for adatoms was found independently by McRae and Petroff (Ref. 10) and by Takayanagi (Ref. 11), but their calculations did not take account of subsurface relaxations which could be significant as shown by Tromp (Ref. 14). 


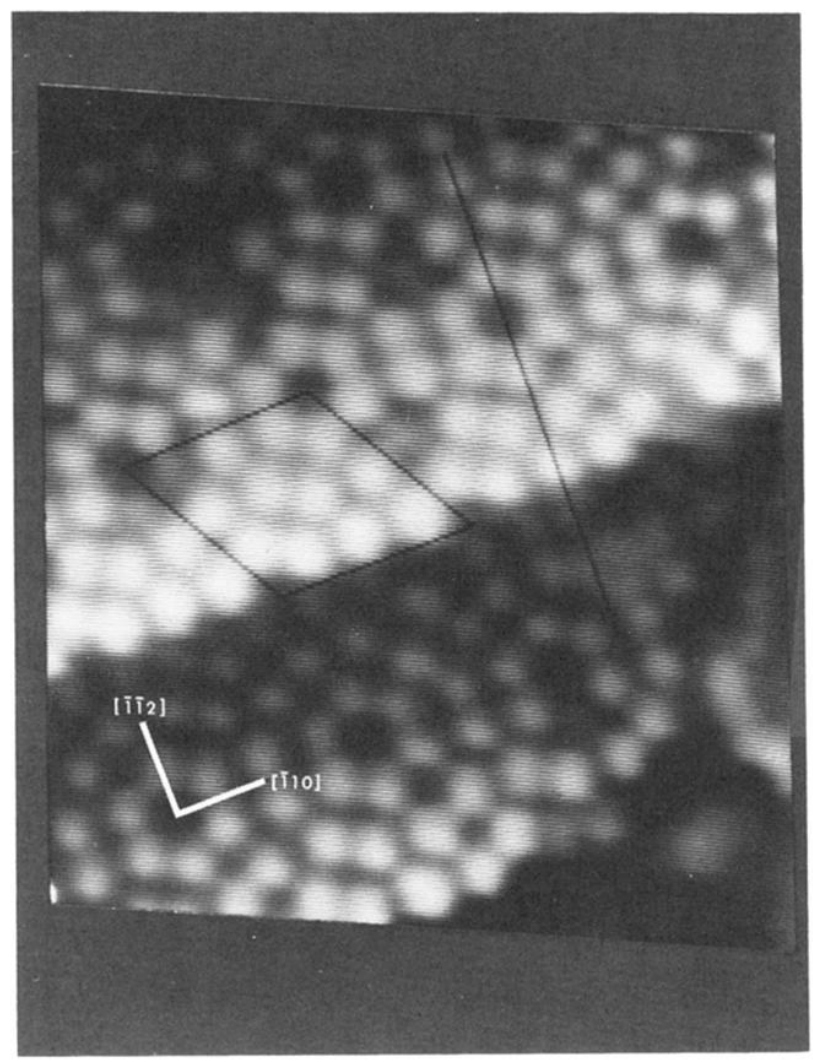

FIG. 2. The data in Fig. 1 displayed as a gray-scale plot. A unit mesh on the edge of the upper step is outlined. The length of one side of the unit mesh is $26.9 \AA$. The straight line shows the tip trajectory mentioned in the text. 


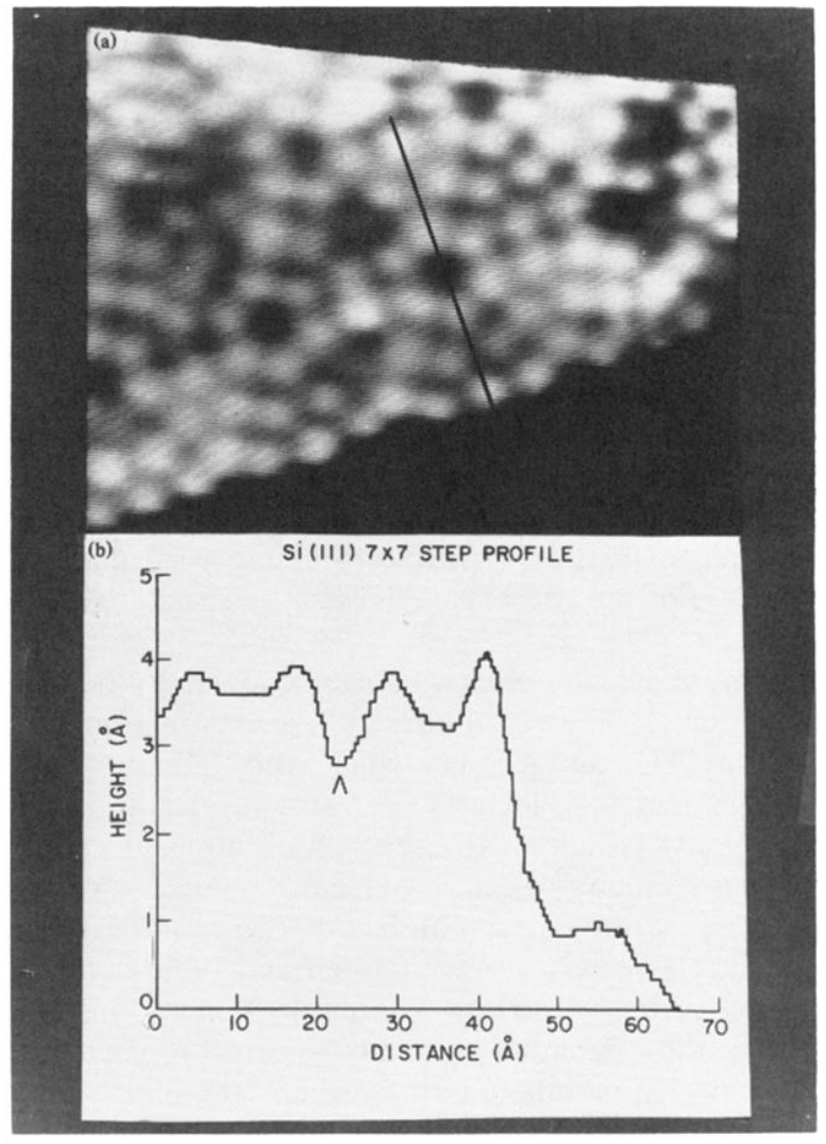

FIG. 3. (a) A tunneling image of the upper step, taken at $-3-\mathrm{V}$ bias. The asymmetry between the two halves of the unit mesh is clearly visible. (b) A plot of the data along the trajectory which bisects the different halves of a unit mesh. The arrow points to the unit-mesh corner hole. 


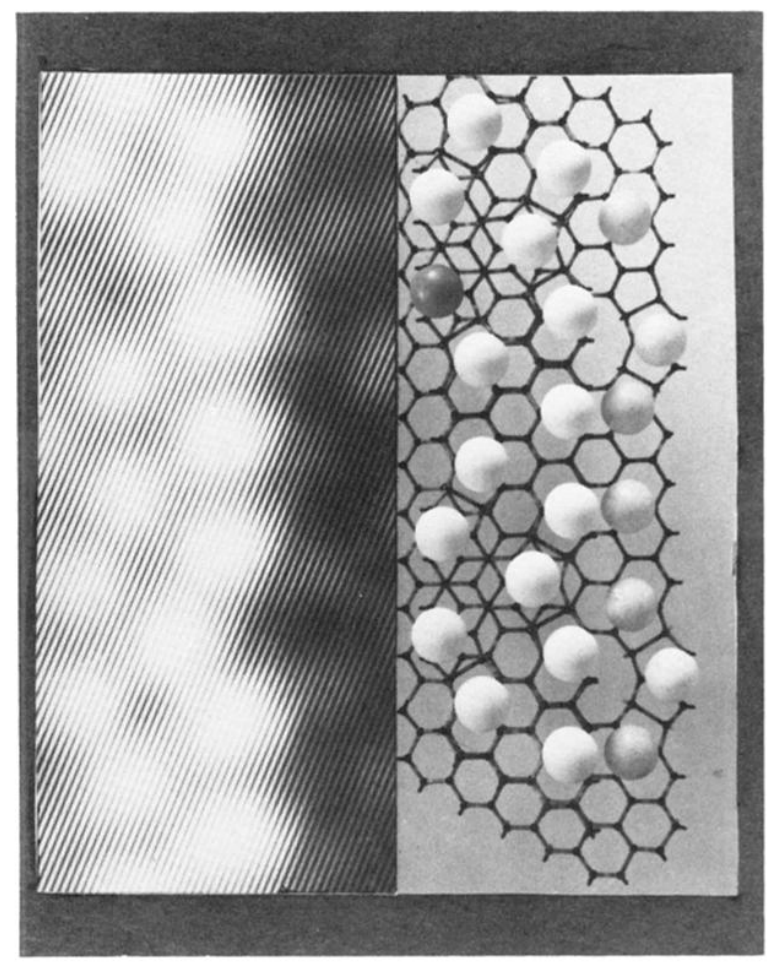

FIG. 4. A portion of data along the upper step edge (from Fig. 2) shown with a model of the same area in which the terraces have the DAS structure. 